\title{
Influence of exposure time to saliva and antioxidant treatment on bond strength to enamel after tooth bleaching: an in situ study
}

\author{
Thais Aglaet Matos MIRANDA ${ }^{1}$, Sandra Kiss MOURA², Vitor Hugo de Oliveira AMORIM ${ }^{3}$, Raquel Sano Suga TERADA ${ }^{1}$, \\ Renata Corrêa PASCOTTO ${ }^{1}$
}

\author{
1- Department of Dentistry, State University of Maringá, Maringá, PR, Brazil. \\ 2- Department of Dentistry, University North of Paraná (UNOPAR), Londrina, PR, Brazil. \\ 3- Private practice, Maringá, PR, Brazil
}

Corresponding address: Renata Corrêa Pascotto - Av. Mandacaru, 1550 - bloco S/08 - 87.080-000 - Maringá - PR - Brasil - Phone: +55 $449982-0215$ - +55 44 3011-9051 - e-mail: renatapascotto@gmail.com

Submitted: January 8, 2013 - Modification: June 28, 2013 - Accepted: September 12, 2013

\section{ABSTRACT}

$\mathrm{O}$ bjectives: This study evaluated the influence of different exposure times to saliva in situ in comparison with an antioxidant treatment on composite resin bond strength to human enamel restored after tooth bleaching. Material and Methods: Forty human teeth specimens measuring $5 \times 5 \mathrm{~mm}$ were prepared and randomly allocated into 5 groups with 8 specimens each: Gct (control group, restored on unbleached enamel); Gbl (restored immediately after bleaching); Gsa (bleached, treated with $10 \%$ sodium ascorbate gel for 60 min and restored); G7d (bleached, exposed to saliva in situ for 7 days and restored); and G14d (bleached, exposed to saliva in situ for 14 days and restored). Restored samples were cut into $0.8 \mathrm{~mm}^{2}$ sticks that were tested in microtensile. Specimens were microscopically analyzed and failure modes were classified as adhesive, cohesive, or mixed. Pretest and cohesive failures were not considered in the statistical analysis, which was performed with one-way ANOVA and Tukey's post-hoc test $(\alpha=0.05)$, with the dental specimen considered as the experimental unit. Results: Mean bond strength results found for $\mathrm{Gb}$ in comparison with Gct indicated that bleaching significantly reduced enamel adhesiveness $(P<0.01)$. However, no statistically significant differences were found between Gct, Gsa and G7d $(P>0.05)$. Bond strength found for $G 14 d$ was significantly higher than for Gsa $(P<0.01)$. Fractures modes were predominantly of a mixed type. Conclusions: Bonding strength to bleached enamel was immediately restored with the application of sodium ascorbate and exposure to human saliva in situ for at least 7 days. Best results were obtained with exposure to human saliva in situ for 14 days. Treatment with sodium ascorbate gel for 60 min may be recommended in cases patients cannot wait for at least 7 days for adhesive techniques to be performed.

Key words: Tooth bleaching. Dental enamel. Saliva. Antioxidants. Adhesives.

\section{INTRODUCTION}

The desire for whiter teeth has motivated an increasing number of patients to seek dental offices for tooth-bleaching treatments ${ }^{14}$. Despite the esthetic benefits brought to patients, it has been demonstrated that bleaching agents might have a negative influence on the integrity of organic enamel structures ${ }^{1,20}$. Mineral loss, increased surface roughness, decreased microhardness, decreased fracture toughness, and reduced enamel microtensile strength, which can result in decreased bond strength to enamel by up to $75 \%$ immediately after bleaching, have already been reported ${ }^{1,4,16,20,26}$. Changes in the amount, length, and morphology of resin tags observed in studies using scanning electron microscopy (SEM) suggest decreased penetration of resin materials into bleached enamel ${ }^{29}$. Clinically, this decrease is important as bleaching is often performed as a preliminary esthetic treatment on teeth to be reconstructed 
with adhesive techniques ${ }^{17}$.

Enamel surface alterations after tooth bleaching, such as those mentioned above, have been found to be more pronounced under in vitro than in in situ settings ${ }^{1,11}$. This finding is attributed to the ability of saliva to prevent such alterations in the dental structure ${ }^{1}$, and suggests the reversibility of bleaching-related changes. By placing bleached teeth under the direct influence of human saliva, the effects of salivary antioxidants on enamel can be better observed ${ }^{26}$. Hence, in situ methodologies have been used to estimate the time needed to reverse bleaching-related effects on enamel bonding, and to determine how soon after bleaching teeth can be restored ${ }^{1}$. Some authors have postulated a waiting period ranging from 24 hours to 3 weeks before performing bonding procedures after bleaching ${ }^{5,23,29}$, while others have suggested a waiting period of 7 days for enamel28 and 14 days for dentin ${ }^{2}$. Nevertheless, in some clinical situations, patients may not be able to wait to have their teeth rehabilitated. In order to permit immediate restorations after bleaching, several researchers have attempted the use of different antioxidant agents prior to performing restorative procedures, among which sodium ascorbate has shown promising results ${ }^{12,14,16,27}$.

Therefore, the debate on how to more adequately deal with the different clinical demands of patients who undergo teeth-whitening and their need for rehabilitation afterwards is still open. In order to shed some more light on the issue, the objective of the present comparative study was to evaluate the influence of different exposure times to saliva in situ and a treatment with $10 \%$ sodium ascorbate gel on composite bond strength to human enamel after bleaching.

\section{MATERIAL AND METHODS}

This study was approved by the State University of Maringá Research Ethics Committee (protocol $609 / 2009$ ). Volunteers provided their written informed consent before participating in the study.

\section{Dental specimen preparation}

Fifty sound extracted human teeth (premolars and molars) were cleaned with periodontal curettes and ultrasound, submitted to prophylaxis with pumice and water ${ }^{18}$, and stored in $2 \%$ formaldehyde solution ( $\mathrm{pH} 7.0$ ) between 30 to 50 days. An enamel block specimen measuring $5 \times 5 \mathrm{~mm}$ was obtained from the flattest portion of each tooth (proximal face for premolars and vestibular or proximal face for molars) with a diamond disc (Diamond Wheel 012" x fine, South Bay Technology Inc, CA, USA) adapted to a sectioning machine (Isomet 1000, Buehler, Lake Bluff, IL, USA) at low speed, under water cooling. To facilitate their handling during subsequent stages, specimens were kept with a minimum height of $3.1 \mathrm{~mm}$. After inspection under optical microscopy 20X (BEL MicroImage Analyzer, Bel Photonics, Monza, Milano, Italy), 10 specimens presenting cracks, fractures or stains were excluded from the study.

Before their use in the experiment, specimens were ultrasound-washed with distilled water for 10 min, dried with jets of air, and randomly allocated into 5 experimental groups with 8 specimens each: Gct (unbleached specimens restored with composite

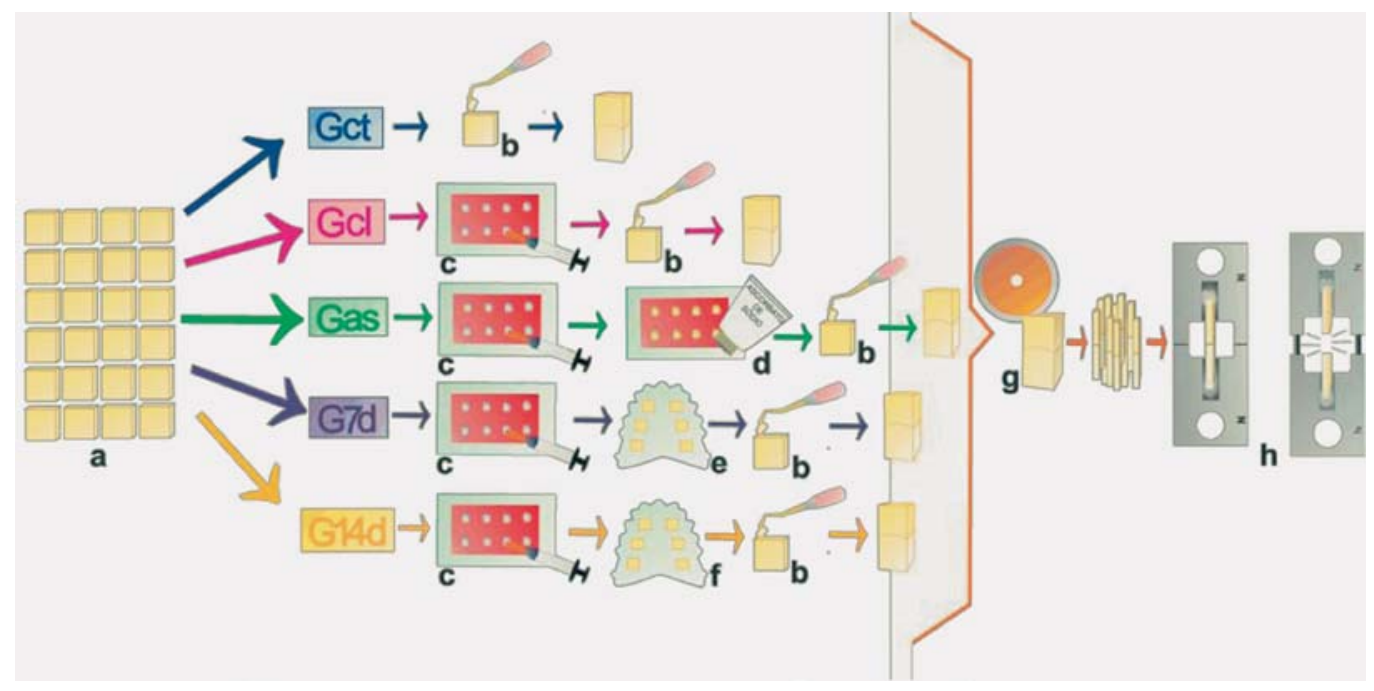

Figure 1- Illustration showing the steps involved in the different experimental procedures: a, dental specimens; b, restoration with composite resin; c, bleaching for $40 \mathrm{~min}$; d, application of sodium ascorbate gel for 60 min; e, placement of bleached dental specimens in intraoral devices to provide contact with saliva in situ for 7 days; $\mathrm{f}$, placement of bleached dental specimens in intraoral devices to provide contact with saliva in situ for 14 days; g, specimens are serially cut for the fabrication of sticks; $h$, sticks are attached to a metal grip for microtensile testing 
resin); Gbl (specimens restored immediately after bleaching); Gsa (bleached specimens treated with sodium ascorbate gel for $60 \mathrm{~min}$ before restoration); G7d (bleached specimens restored after being exposed to saliva in situ for 7 days); and G14d (bleached specimens restored after being exposed to saliva in situ for 14 days). Figure 1 illustrates the steps involved in the different experimental procedures described below.

\section{Bleaching procedure}

Specimens belonging to Gbl, Gsa, G7d and G14d were fixed to a plate of pink dental wax (Cera utilidade rosa Epoxiglass, Epoxiglass Ind. Com. de Produtos Químicos Ltda., Diadema, SP, Brazil) placed on a glass sheet in such a way as to expose the enamel surface. A thin layer $(1 \mathrm{~mm})$ of $37.5 \%$ hydrogen peroxide-based bleaching gel (Pollaoffice +, SDI Limited, Bayswater, Victoria, Australia) was applied over the enamel surface. After 8 minutes, the gel was removed with a fine tipped suction device (Sug-Plast, DFL Indústria e Comércio S.A., Rio de Janeiro, RJ, Brazil) and reapplied. This procedure was repeated 4 times, totaling 5 applications. Specimens were in contact with the product for a total of $40 \mathrm{~min}$, after which they were rinsed with jets of water for 30 seconds, and dried with compressed air.

\section{Treatment with sodium ascorbate}

Still fixed to the plate of pink wax, bleached specimens assigned to Gsa were treated with $10 \%$ sodium ascorbate gel ( $\mathrm{pH} 7)$ manipulated according to Kimyai and Valizadeh ${ }^{14}$ (2006) shortly before its application, and placed into a chamber with $100 \%$ humidity for 60 minutes. After this period specimens were rinsed with jets of water for 30 seconds, and dried with compressed air.

\section{Exposure to saliva in situ}

To evaluate the effect of exposure time to human saliva on composite bond strength to bleached enamel, 3 volunteers (two female and one male) were selected. The inclusion criteria were normal salivary flow, absence of caries or periodontal diseases, non-pregnant women, and absence of systemic disorders; while the exclusion criteria were smokers and current use of orthodontic appliances or fixed or removable dentures. Alginate impressions (Jeltrate Dustless, Dentsply, York, PA, USA) of the maxillary arches were taken, and plaster models (Durone IV, Dentsply, York, PA, USA) were prepared for each volunteer. Polyvinyl siloxane blocks (Elite $\mathrm{HD}+$, Zhermack Clinical, Badia Polesine, Rovigo, Italy) measuring $5.5 \times 5.5 \times 5.5 \mathrm{~mm}$ were bonded to the palatal area of the plaster models with a cyanoacrylate-based glue (Super Bonder, Loctite, São Paulo, SP, Brazil). The plaster models were then isolated and intraoral palatal devices were fabricated in acrylic resin (JET, Clássico Artigos Odontológicos Ltda., Campo Limpo Paulista, SP, Brazil). The 16 specimens to be assessed in situ (G7d and G14d) were fixed with sticky wax (Cera utilidade Asfer, ASFER - Indústria Química Ltda., São Caetano do Sul, SP, Brazil) to the cavities left by the polyvinyl siloxane blocks in the intraoral palatal devices ( 5 in two models and 6 in one). To make sure that bleached specimens would be in clear contact with the oral cavity, they were fixed in such a way that the enamel surfaces would coincide with that of the acrylic device.

Each volunteer received their palatal device and were instructed to use it throughout the day and night. It could only be removed during meals, when ingesting liquids (except water), or when cleaning their teeth. When removed from the mouth, the devices were stored in a container with gauze imbibed in distilled water. Volunteers were instructed to clean their palatal devices under running water only, and to avoid any contact with fluoride-containing solutions. On the morning of the eighth day, eight specimens (G7d) were randomly removed from the palatal devices (2, 3 and 3 ), and the cavities filled with sticky wax. The devices were then returned to the volunteers, who wore the palatal devices until the morning of the $15^{\text {th }}$ day, when they were returned and the remaining specimens (G14d) removed. After exposure to saliva, specimens were rinsed with jets of water for 30 seconds, and dried with compressed air.

\section{Restoration with composite resin}

Before being restored, all specimens were measured with a thickness gauge (Espessímetro Otto inox, Arminger \& Cia Ltda, São Leopoldo, RS, Brazil). The enamel surface of specimens was treated with $37 \%$ phosphoric acid etching for 30 $\mathrm{sec}$ and rinsed under running water for $30 \mathrm{sec}$. After that, two layers of an adhesive system (Adper Single Bond 2, 3M-ESPE, 3M do Brasil, Campinas, $\mathrm{SP}$, Brazil) were applied on the enamel surface, which was light activated for 20 seconds after excess had been removed with the application of jets of air for 5 seconds. Composite resin (Filtek Z350, 3M-ESPE, 3M do Brasil, Campinas, SP, Brazil) was then placed in increments of $1 \mathrm{~mm}$ and light activated $\left(600 \mathrm{~mW} / \mathrm{cm}^{2}\right)$ with an halogencuring device (Optilux 501 - Demetron, Kerr, West Collins, CA, USA) for 20 seconds. The restoration was considered concluded when the height of the restoration coincided with the initial height of the specimen. Final heights ranged from 6.2 to $10 \mathrm{~mm}$. After restoration, specimens were stored in distilled water at $37^{\circ} \mathrm{C}$ for $24 \mathrm{~h}$. 


\section{Microtensile testing}

Each individual specimen was removed from distilled water, jet dried and fixed onto the acrylic brackets of a precision sectioning machine (Isomet 1000, Buehler, Lake Bluff, IL, USA) with sticky wax. Specimens were then serially sectioned (Extec Corp. 12205) perpendicularly through the tooth/ restoration interface into $0.9 \mathrm{~mm}$ slices. Each slice was then individually fixed to the cutting machine to be further sectioned as to obtain tooth/restoration "sticks" with a cross-sectional area of approximately $0.8 \mathrm{~mm}^{2}$. Measurements were performed with a digital caliper (Zaas Precision, Amatools, Piracicaba, $\mathrm{SP}$, Brazil). After sectioning, sticks were stored at $100 \%$ relative humidity to avoid desiccation before testing.

Each stick was placed in a special microtensile jig (Odeme Biotechnology, Joaçaba, SC, Brazil) and the extremities were completely covered with cyanoacrylate glue (adhesive ZAP and Zip Kicker accelerator, Colas, Pacer Technology, USA), leaving just the enamel/resin interface exposed. This was done to make sure the stick was correctly centralized on the device and tension distribution was concentrated at the interface. Sticks were tensile-tested at $0.5 \mathrm{~mm} / \mathrm{min}$ in a universal testing machine (EMIC DL 2000, São José dos Pinhais, $\mathrm{PR}$, Brazil) until failure. Tensile force in $\mathrm{N}$ was divided by the cross-sectional area in $\mathrm{mm}^{2}$ and bond strength values were expressed in $\mathrm{MPa}$. To determine whether a specimen would be included in the statistical analysis, fragments were observed under an optical microscope 40X (Bel MicroImage Analyzer, Bel Photonics, Monza, Italy), and fracture types were classified as follows ${ }^{7}$ :

Adhesive fracture - failure in adhesion, with fracture at the interface;

Cohesive fracture - enamel substrate failure only;

Cohesive fracture - restorative material failure only; or

Mixed fracture - enamel substrate and resin materials fractures (adhesive or resin composite) in the same test specimen.

Sticks that presented pretest failures, i.e., broke during preparation for the test, were discarded from the analysis ${ }^{21}$. Moreover, in order to confirm the findings from the optical microscopy, $30 \%$ of each sample was randomly selected for interface analysis under scanning electron microscopy (SEM). Sticks were placed in aluminum stubs, sputtercoated with gold-palladium alloy (Ion Coater, IC50, Shimadzu Biotech), and observed under SEM (SS-550 Superscan, Shimadzu Biotech, Japan). Only sticks with "adhesive" or "mixed" interface fractures, in which the cohesive part represented $\leq 10 \%$ of the interface area, were included in the statistical analysis ${ }^{10,25}$.

\section{Statistical analysis}

To guarantee independence of data, and at the same time reduce the variability, average microtensile strength was calculated for the sticks originating from each dental specimen ${ }^{21}$. As the results presented normal distribution, they were statistically analyzed with one-way analysis of variance (ANOVA) and Tukey's post-hoc test, at 5\% global significance.

\section{RESULTS}

The cross-sectional areas of sticks were statistically homogenous $\left(0.70-0.92 \mathrm{~mm}^{2}, \mathrm{P}=0.52\right)$, indicating that differences in bond strength among groups would not be due to differences in the crosssectional area.

Mean ( $\pm S D$ ) bond strength found were: Gct 39.61 ( \pm 13.71$)$, Gbl 25.89 ( \pm 11.38$)$, Gsa 36.27 ( \pm 16.04$),$ G7d 43.60 ( \pm 15.24$)$, and G14d 49.26 ( \pm 12.97$) \mathrm{MPa}$ (Figure 2). Results found for $\mathrm{Gbl}$ in comparison to Gct indicate that bleaching significantly reduced enamel adhesiveness $(P<0.01)$. However, no statistically significant differences were found between Gct, Gsa and G7d $(P>0.05)$, demonstrating that the treatment with sodium ascorbate and exposure to saliva for 7 days recovered bond strength to enamel to the same level of unbleached specimens. Although bond strength found for G14d was statistically the same as for Gct and G7d, it was significantly higher than that found for Gsa $(P<0.01)$.

Table 1 illustrates premature failures and fracture mode ${ }^{15}$ observed in the different experimental groups. Qualitative microscopic analysis (optical and SEM) revealed that fractures modes (Figures $3-4)$ were predominantly of a mixed type (52\%), followed by adhesive (28\%) and cohesive (20\%). The frequency of premature failures was highest in

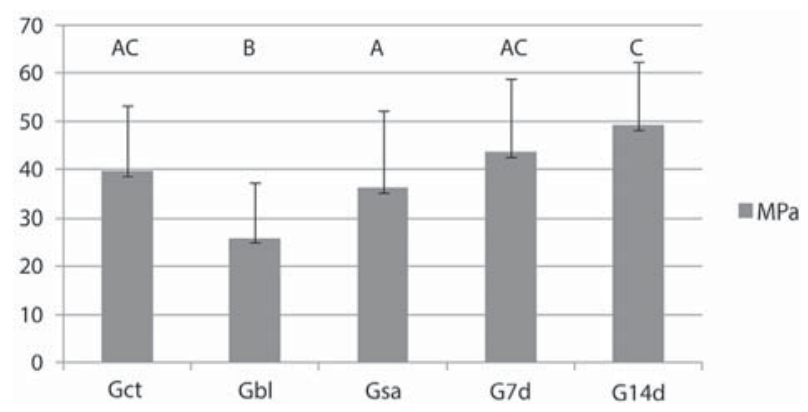

Figure 2- Mean bond strength ( $\mathrm{MPa}$ ) and standard deviation found in the microtensile testing. Gct: control group; Gbl: group restored immediately after bleaching; Gsa: group restored after bleaching and treatment with sodium ascorbate; G7d: group restored after 7 days in human saliva; and G14d: group restored after 14 days in human saliva. Similar letters indicate no significant differences between groups (Tukey's test; P>0.05) 
Table 1- Premature failures and fracture modes observed in the experimental groups, $\mathrm{N}(\%)$

\begin{tabular}{cccccc}
\hline Group & $\begin{array}{c}\text { Premature } \\
\text { failures }\end{array}$ & $\begin{array}{c}\text { Cohesive } \\
\text { fractures }\end{array}$ & $\begin{array}{c}\text { Adhesive } \\
\text { fractures }\end{array}$ & Mixed fractures & $\begin{array}{c}\text { Total number of } \\
\text { sticks }\end{array}$ \\
\hline Gct & $13(18 \%)$ & $13(18 \%)$ & $13(18 \%)$ & $33(46 \%)$ & 72 \\
Gbl & $60(78 \%)$ & $6(7 \%)$ & $9(12 \%)$ & $2(3 \%)$ & 77 \\
Gsa & $8(11 \%)$ & $10(14 \%)$ & $16(22 \%)$ & $39(53 \%)$ & 73 \\
G7d & $16(23 \%)$ & $9(13 \%)$ & $13(19 \%)$ & $31(45 \%)$ & 69 \\
G14d & $13(18 \%)$ & $12(16.7 \%)$ & $19(26.4 \%)$ & $28(38.9 \%)$ & 72 \\
Total & $110(30.3 \%)$ & $50(19.8 \%)$ & $70(27.7 \%)$ & $133(52.5 \%)$ & 363 \\
\hline
\end{tabular}

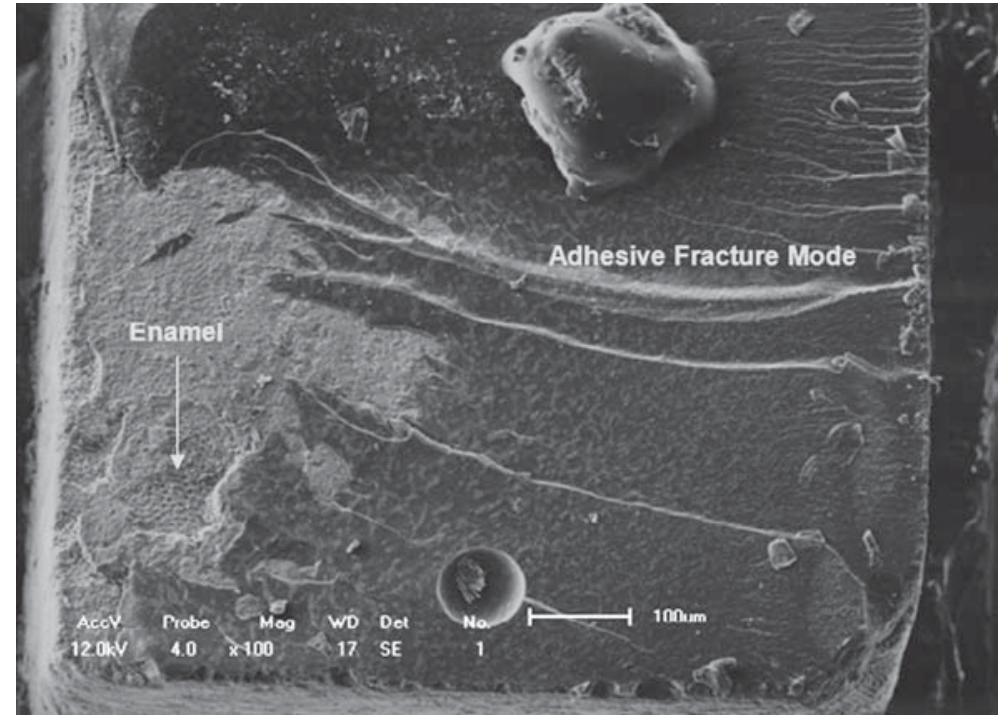

Figure 3- Interface of a test specimen from G7d seen with scanning electron microscopy. Typical fracture mode was classified as mixed (adhesive+cohesive in enamel). Arrow points to the fracture region in enamel $(B a r=100 \mu \mathrm{m})$

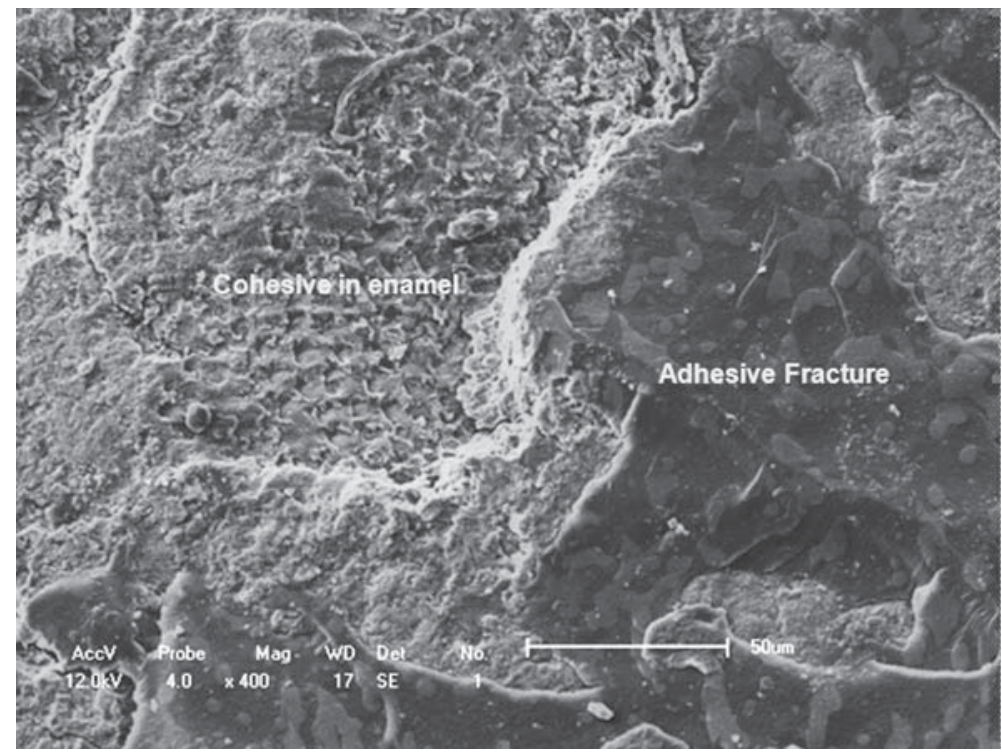

Figure 4- Interface of the same test specimen as shown in Figure 3, at higher magnification. Note the adhesive and cohesive fractures in enamel $($ Bar $=50 \mu \mathrm{m})$ 
the Gbl group (78\%), followed by G7d (23\%), Gct and G14d (both $18 \%$ ), and Gsa (11\%).

\section{DISCUSSION}

To the best of our knowledge, this is the first report to evaluate the influence of exposure time to saliva in situ (natural antioxidant effect) in comparison to a treatment with $10 \%$ sodium ascorbate gel (artificial antioxidant effect) on composite resin bond strength to human enamel after bleaching.

The results found for $\mathrm{Gbl}$ in comparison with Gct confirmed that the treatment with hydrogen peroxide reduced enamel adhesiveness in approximately $35 \%$, which is consistent with previous results $1,4,16,20,26$. This finding may be explained by the delayed release of oxygen due to the presence of peroxides and their subproducts, oxygen and water, which compete with free radicals produced during the photoactivation ${ }^{17}$, affecting the

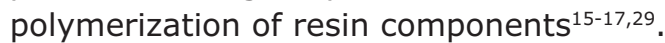

In the present study, the treatment with sodium ascorbate increased composite resin bonding to enamel immediately after bleaching. This finding is in agreement with previous studies that have shown antioxidants to be able to immediately restore bond strength to bleached enamel $12,14,27$. However, Kaya, et al. ${ }^{12}$ (2008) suggested that to obtain effective results the exposure time to the antioxidant agent should be of at least $60 \mathrm{~min}$. A similar protocol was used in the present study (Gsa), with satisfactory results. In another study, Torres, et al. ${ }^{26}$ (2006) observed that the treatment of dental specimens with a sodium ascorbate solution for 20 minutes was not sufficient to restore bond strength to bleached enamel. The authors' findings may have not only been due to insufficient exposition to the antioxidant agent, but also to the way it was conducted. In the present study, the sodium ascorbate agent used was in a gel form, which is easy to apply and, according to some studies ${ }^{12,27}$, more effective than solutions, as the active compounds in the gel are released more slowly.

Although an in vitro study has demonstrated that the amount of sodium ascorbate required to reduce hydrogen peroxide is directly related to the concentration of the latter, and the reaction kinetics between oxidant and antioxidant showed that 5 minutes was sufficiently long for the antioxidant to exert an effect ${ }^{8}$, the exact time required for the antioxidant to reestablish bond capacity to bleached enamel in vivo has yet to be established. Lai, et al. ${ }^{16}$ (2002) recommended the use of $10 \%$ sodium ascorbate for at least a third of the bleaching agent application time. Following this recommendation, Kimyai and Valizadeh ${ }^{14}$ (2006) left the antioxidant in contact with bleached teeth for 3 hours, which was considered too long from a clinical perspective. Studies have also reported differences in the effective application time between carbamide peroxide and hydrogen peroxide. Studies using 10\% carbamide peroxide showed that bonding strength to enamel was restored within 10 to 180 minutes $^{13}$ or 10 to 480 minutes $^{12}$ after the application of a sodium ascorbate gel and solution, respectively. Whereas, for enamel bleached with 35\% hydrogen peroxide, the effective reversal of reduced bonding was achieved after $10-20$ minutes $^{12,26}$ of exposure to a $10 \%$ sodium ascorbate solution.

Sazaki, et al. ${ }^{24}$ (2009) attempted to simulate home-bleaching using a tray and artificial saliva. Bond strength to bleached enamel was not restored by the treatment with $10 \%$ sodium ascorbate solution, perhaps due to the fact that the antioxidant was diluted by the artificial saliva in which it was incubated for 2 hours. In the present study, dental specimens with the gel were kept in an environment at $100 \%$ humidity without any contact with fluid. This scenario was used to reproduce the clinical application of the antioxidant in a dental office, which is performed under relative isolation. Thus, the sodium ascorbate gel used was not dissolved or diluted, which may have improved its ability to restore bond strength to enamel more effectively.

Therefore, in the present study, the treatment of bleached enamel surface with $10 \%$ sodium ascorbate antioxidant gel for $60 \mathrm{~min}$ was found to be an effective clinical option for patients in need of immediate esthetic restorations following bleaching. In a follow-up study performed by Garcia, et al. ${ }^{9}$ (2012), the authors reported on restorations carried out immediately after bleaching with the use of $10 \%$ sodium ascorbate gel for one hour. The study demonstrated that no alterations in restoration shape or color were observed after one year, and considered the method efficient, user-friendly and little time-consuming. One of the limitations of using sodium ascorbate as an antioxidant, however, is that it gradually oxidizes with time, becoming less reductive ${ }^{19}$. To circumvent this problem in the present study, the sodium ascorbate gel used was manipulated shortly before its application. Nevertheless, further investigations are needed to determine its shelf life.

No statistical differences between Gct, G7d, and G14d groups were found, indicating that bond strength to enamel was fully reestablished after exposure to saliva for 7 days in situ (Figure 2). Although several authors have recommended that restorative procedures should wait between 7 to 14 days after bleaching $3,12,15,17,23,26,29$, the findings in this study suggest that exposition to saliva for at least one week is a satisfactory waiting period for restorative procedures after in-office dental bleaching treatments. However, different 
hydrogen peroxide concentrations can also influence the waiting time required between the end of the bleaching treatment and the performance of bonding procedures ${ }^{3}$. Studies using hydrogen peroxide have suggested a waiting period of 14 days after bleaching before starting with the restorative procedure. The results of the present study support this assumption, as bond strength to bleached enamel after being exposed for 14 days (G14d) to human saliva was significantly higher than that found for sodium ascorbate-treated enamel (Gsa).

Results obtained in laboratory studies may differ from the clinical situation, because they commonly use artificial saliva to store the specimens ${ }^{3}$. In situ studies have the advantage of being nearest to the clinical situation, as bleached dental specimens are placed under the direct effect of human saliva ${ }^{5}$. Human saliva acts on the oxidative stress mediated by free radicals, and presents alternative antioxidants, including ascorbic acid and vitamin $\mathrm{E}^{10}$. A previous in situ and in vitro study has shown that although bleaching agents are capable of altering dental enamel surface's microhardness, roughness, and morphology, natural saliva allows for enamel mineral reposition ${ }^{20}$. This process helps to reestablish enamel surface microhardness, which is an important component in the adhesive strength of enamel ${ }^{22}$. In an attempt to simulate the clinical situation, as well as to permit that only the natural antioxidant effect of saliva could be assessed in the reestablishment of adhesive bond strength after bleaching, volunteers in the in situ experiment were asked to avoid the use of any type of fluoridecontaining products. These products have been shown to alter salivary fluoride concentration ${ }^{6}$, which could have had an influence on enamel's remineralization process.

In this study, $52 \%$ of fractures were of the mixed type, while $28 \%$ were adhesive. This means that resin-enamel interfaces were tested in tensile as expected. The frequency of premature failures in the Gbl group was much higher (78\%) than that observed in other groups. During the specimen preparation procedures, microcracks might have been inadvertently produced mainly by the vibrations of the cutting instruments. Such defects, coupled with the brittle nature of enamel, are responsible for the premature failure of microtensile bond specimens ${ }^{7}$. The high number of pretest failures seen in the Gbl group emphasizes the fragility of bonding immediately after bleaching. In order to standardize procedures and avoid variability as much as possible, we followed the recommendations set out by Roulet and Van Meerbeek $^{21}$ (2007). Pretest failures were ignored and not used in the statistical analysis. SEM was used to analyze in detail the type of failure, and only those sticks presenting adhesive or predominantly adhesive failures were considered, while cohesive failures were discarded as well. This was done to circumvent the inherent problems with the microtensile tests mentioned by El Zohairy, et al. ${ }^{7}$ (2010), concerning the high presence of cohesive fractures. Moreover, to minimize the differences in adhesion that could occur in different parts of the specimen, we calculated the average microtensile bond strength for the sticks originating from the same dental specimen.

In conclusion, based on the results found in this study, the use of sodium ascorbate after in-office bleaching may be indicated in clinical situations in which the patient cannot wait for adhesive techniques to be performed. However, whenever possible, bonding should be postponed for at least 7 days after tooth bleaching. From a chemical-molecular perspective, the alterations in the bleached enamel caused by the antioxidant treatment and exposure to saliva remain unknown and warrant further investigation.

\section{CONCLUSIONS}

1. Composite resin bond strength to enamel was reduced by bleaching.

2. When $10 \%$ sodium ascorbate gel was applied to the bleached enamel for 60 minutes, bond strength was reverted to the same level found for unbleached enamel.

3. When bleached enamel was exposed to human saliva for 7 days, bond strength was reestablished to the same level of enamel treated with $10 \%$ sodium ascorbate gel and unbleached enamel.

4. When bleached enamel was exposed to human saliva in situ for 14 days, bond strength was significantly better than that found for specimens treated with $10 \%$ sodium ascorbate gel.

\section{REFERENCES}

1- Attin T, Schmidlin PR, Wegehaupt F, Wiegand A. Influence of study design on the impact of bleaching agents on dental enamel microhardness: a review. Dent Mater. 2009;25:143-57.

2- Barbosa CM, Sasaki RT, Florio FM, Basting RT. Influence of time on bond strength after bleaching with $35 \%$ hydrogen peroxide. J Contemp Dent Prac. 2008;9:81-8.

3- Bittencourt ME, Trentin MS, Linden MS, Oliveira Lima Arsati YB, França FM, Flório FM, et al. Influence of in situ postbleaching times on shear bond strength of resin-based composite restorations. J Am Dent Assoc. 2010;141:300-6.

4- Cavalli V, Giannini M, Carvalho RM. Effect of carbamide peroxide bleaching agents on tensile strength of human enamel. Dent Mater. 2004;20:733-9.

5- Dishman MV, Covey DA, Baughan LW. The effects of peroxide bleaching on composite to enamel bond strength. Dent Mater. 1994; 10:33-6.

6- Duckworth RM, Maguire A, Omid N, Steen IN, McCracken GI, Zohoori FV. Effect of rinsing with mouthwashes after brushing with a fluoridated toothpaste on salivary fluoride concentration. Caries Res. $2009 ; 43(5): 391-6$. 
7- El Zohairy AA, Saber MH, Abdalla AI, Feilzer AJ. Efficacy of microtensile versus microshear bond testing for evaluation of bond strength of dental adhesive systems to enamel. Dent Mater. 2010;26:848-54.

8- Freire A, Souza EM, Menezes Caldas DB, Rosa EA, Bordin CF, Carvalho RM, et al. Reaction kinetics of sodium ascorbate and dental bleaching gel. J Dent. 2009;37:932-6.

9- Garcia EJ, Mena-Serrano A, Andrade AM, Reis A, Grande RH, Loguercio $A D$. Immediate bonding to bleached enamel treated with $10 \%$ sodium ascorbate gel: a case report with one-year follow-up. Eur J Esthet Dent. 2012;7(2):154-62.

10- Gümüş $P$, Buduneli N, Cetinkalp S, Hawkins SI, Renaud D, Kinane DF, et al. Salivary antioxidants in patients with type 1 or type 2 diabetes and inflammatory periodontal disease: a casecontrol study. J Periodontol. 2009;80:1440-6.

11- Justino LM, Tames DR, Demarco FF. In situ and in vitro effects of bleaching with carbamide peroxide on human enamel. Oper Dent. 2004;29:219-25.

12- Kaya $A D$, Türkün M, Arici M. Reversal of compromised bonding in bleached enamel using antioxidant gel. Oper Dent. 2008;33:441-7.

13- Kimyai S, Oskoee SS, Rafighi A, Valizadeh H, Ajami AA, Helali ZN. Comparison of the effect of hydrogel and solution forms of sodium ascorbate on orthodontic bracket-enamel shear bond strength immediately after bleaching: an in vitro study. Indian J Dent Res. 2010;21:54-8.

14- Kimyai S, Valizadeh $\mathrm{H}$. The effect of hydrogel and solution of sodium ascorbate on bond strength in bleached enamel. Oper Dent. $2006 ; 31: 496-9$.

15- Lago AD, Freitas PM, Netto NG. Evaluation of the bond strength between a composite resin and enamel submitted to bleaching treatment and etched with Er:YAG laser. Photomed Laser Surg. 2011;29:91-5.

16- Lai SC, Tay, FR, Cheung GS, Mak YF, Carvalho RM, Wei SH, et al. Reversal of compromised bonding in bleached enamel. J Dent Res. 2002;81:477-81.

17- Miguel LC, Baratieri LN, Monteiro S Jr, Ritter AV. In situ effect of $10 \%$ carbamide peroxide on resin-dentin bond strengths: a novel pilot study. J Esthet Restor Dent. 2004;16:235-42.
18- Moura SK, Pelizzaro A, Dal Bianco K, Goes MF, Loguercio $A D$, Reis $A$, et al. Does the acidity of self-etching primers affect bond strength and surface morphology of enamel? J Adhes Dent. 2006;8(2):75-83.

19- Muraguchi K, Shigenobu, S. Suzuki S, Tanaka T. Improvement of bonding to bleached bovine tooth surfaces by ascorbic acid treatment. Dent Mater J. 2007;26:875-81.

20- Pinto CF, Oliveira R, Cavalli V, Gianninni M. Peroxide bleaching agent effects on enamel surface microhardness, roughness and morphology. Braz Oral Res. 2004;18:306-11.

21- Roulet JF, Van Meerbeek B. Editorial: Statistics: a nuisance, a tool, or a must? J Adhes Dent. 2007;9:287-8.

22- Sa Y, Sun L, Wang Z, Ma X, Liang S, Xing W, et al. Effects of two in-office bleaching agents with different $\mathrm{pH}$ on the structure of human enamel: an in situ and in vitro study. Oper Dent. 2013;38(1):100-10.

23- Santana FR, Pereira JC, Pereira CA, Fernandes Neto AJ, Soares $\mathrm{CJ}$. Influence of method and period of storage on the microtensile bond strength of indirect composite resin restorations to dentine. Braz Oral Res. 2008;22:352-7.

24- Sasaki RT, Flório FM, Basting RT. Effect of $10 \%$ sodium ascorbate and $10 \%$ alpha-tocopherol in different formulations on the shear bond strength of enamel and dentin submitted to a home-use bleaching treatment. Oper Dent. 2009;34:746-52.

25- Scherer SS, Cesar PF, Swain MV. Direct comparison of the bond strength results of the different test methods: a critical literature review. Dent Mater. 2010;26:e78-93.

26- Torres CR, Koga AF, Borges AB. The effects of anti-oxidant agents as neutralizers of bleaching agents on enamel bond strength. Braz J Oral Sci. 2006;5:971-6.

27- Türkün M, Çelik EU, Kaya AD, Arici M. Can the hydrogel form of sodium ascorbate be used to reverse compromised bond strength after bleaching? J Adhes Dent. 2009;11:35-40.

28- Unlu N, Cobankara FK, Ozer F. Effect of elapsed time following bleaching on the shear bond strength of composite to enamel. J Biomed Mater Res B Appl Biomater. 2008;84:363-8.

29- Wilson D, Xu C, Hong L, Wang Y. Effects of different preparation procedures during tooth whitening on enamel bonding. J Mater Sci Mater Med. 2009;20:1001-7. 